\title{
Steroids in paediatric heart surgery: eminence or evidence-based practice?
}

\author{
Daniel Fudulu $^{1,2} \cdot$ Stafford Lightman $^{1} \cdot$ Massimo Caputo $^{3} \cdot$ Gianni Angelini $^{1}$
}

Received: 2 January 2018 / Revised: 5 March 2018 / Accepted: 8 March 2018 / Published online: 3 April 2018

(C) The Author(s) 2018

\begin{abstract}
Steroids in paediatric heart surgery are given prophylactically to blunt the systemic inflammatory response induced by the extracorporeal circuit and to improve clinical outcomes. However, there is an ongoing controversy about the impact of steroids on clinical outcomes after paediatric heart surgery. The hypothalamic-pituitary-adrenal axis is the primary neuroendocrine system activated during the stress of surgery. Relative adrenal insufficiency can accompany paediatric heart surgery; therefore, perioperative steroid supplementation is still administered by some centres. The studies that investigate the hypothalamic-pituitaryadrenal axis physiology during surgery have many limitations, and it is unclear how to define what is adrenal insufficiency. In this review, we focus on discussing the available evidence for steroid use in paediatric cardiac surgery.
\end{abstract}

Keywords Steroids $\cdot$ Clinical outcomes $\cdot$ Relative adrenal insufficiency

\section{Background}

Most of the progress in cardiac surgery results from the discovery of heparin followed by the introduction of the cardiopulmonary by-pass machine that made possible open cardiac surgery [1]. However, quite early after its introduction in the 1950s, attempts were made to both understand and modulate the adverse effects of the extracorporeal circuit. Indeed, the first study of the effect of glucocorticoids in cardiac surgery was published by Replodge et al. in 1966. The authors looked at the effect of large doses of dexamethasone administered perioperatively in 20 children and adults undergoing heart surgery with the use of cardiopulmonary by-pass [2]. They found steroid recipients to have a lower beta-glucuronidase enzyme activity thus suggesting reduced lysosomal injury. However, there was no clear benefit in terms of clinical

Daniel Fudulu

daniel.fudulu@bristol.ac.uk

1 Department of Cardiac Surgery, Bristol Heart Institute, Bristol, UK

2 Henry Wellcome Laboratories for Integrative Neuroscience and Endocrinology, University of Bristol, Dorothy Hodgkin Building, Bristol, UK

3 Department of Congenital Cardiac Surgery, Bristol Children's Hospital, Bristol, UK outcomes apart from noticing that the steroid recipient patients had a "better general appearance and state postoperatively". Our current understanding of the response to surgery is that the contact of the blood with the extracorporeal circuit, the ischaemia-reperfusion injury, and endotoxemia induce a generalised immune system activation called the systemic inflammatory response. It is also believed that there is a balance between "good" inflammation that acts to prime the immune system and "bad" inflammation that could result in multiple end-organ failure and death [3]. In children, the surgical inflammatory response tends to be amplified due to their lower circulating blood volume compared to the size of the extracorporeal circuit and due to the frequent use of deep hypothermic arrest surgery and haemodilution [4]. According to several surveys of clinical practice, glucocorticoids are still widely used as a pharmacologic tool to modulate the inflammation [5]. Most of the centres do not administer steroids in every case but reserve their use for the most "high-risk" cases: neonates, deep hypothermic cardiac arrest surgery, and long cardiopulmonary by-pass [5]. Such indications vary widely between centres, and there is no consensus because of limited research in this area. Apart from this generalised, immune response to surgery, the stress of surgery is also accompanied by a neuroendocrine response. The primary neuroendocrine system activated during surgery is the hypothalamic-pituitaryadrenal axis (HPA) that produces the stress hormone cortisol. Therefore, another justification behind the use of steroids is to 
cover for the presumed perioperative relative adrenal insufficiency that can accompany cardiac surgery [6]. Finally, steroids are also administered to protect against brain injury during DHCA surgery. In the current review, we will discuss these three main areas of steroid use (Fig. 1). Another indication for steroid administration is immunosuppression after paediatric heart transplantation, but this area is not within the scope of this review.

\section{Methodology}

We conducted a literature search using PubMed and MEDLINE databases. The search phrase included a combination of the following terms: "steroid", "glucocorticoid", "corticosteroid", "dexamethasone", "hydrocortisone", "methylprednisolone", "pediatric", "paediatric", "heart surgery", "cardiac surgery", "children", "neonates", "deep hypothermic circulatory arrest", "adrenal". The last search was conducted in November 2017. We identified additional articles by cross-referencing from author reference lists and published review papers. We have included all relevant articles assessing the effect of corticosteroids on inflammation, clinical outcomes, adrenal function, and brain injury during paediatric heart surgery with the use of cardiopulmonary bypass.

\section{Less inflammation, better clinical outcomes?}

Throughout the years, several studies measured the effect of glucocorticoids on markers of inflammation but most importantly tried to correlate the effect on clinical outcomes. Some studies found that steroids can blunt inflammation [7-14] while others found the opposite [15]. Most importantly, it is unclear to what degree this presumed "anti-inflammatory" effect also translates into better clinical outcomes [7, 10, 13]. Firstly, it is very difficult to characterise the multifaceted

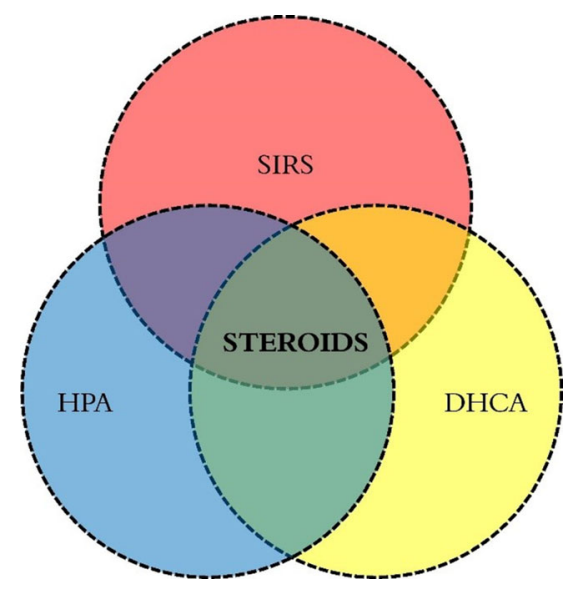

Fig. 1 The main steroid indications in paediatric heart surgery nature of the SIRS response whereby a variety of cytokines are activated and interact in a rather complex, unpredictable manner [16]. Secondly, we know that the use of steroids is subject to huge variability in the practice between centres and this is also reflected in the design of the studies that use various types of steroids, various regimens, or routes of administration thus making more difficult the correlation between inflammation and clinical outcomes $[5,17]$. Thirdly, the available studies are small-sized and display a variability between the endpoints measured (e.g. inflammation or clinical outcomes measured as a primary or the secondary outcome). Finally, it is likely that a standard corticosteroid regimen given to all patients might not address the individual, host inflammatory response and it is likely that the approach to SIRS will have to be personalised rather one-for-all regimen [18].

There are several small size, randomised trials of steroids versus placebo conducted on children of various ages or just neonates [8-10, 19-21]. Their results are conflictive with some trials reporting benefit $[8,11,19,21]$ while others reporting no effect on clinical outcomes $[7,9,13,20]$. The results of an ongoing trial on 190 neonates are still awaited and hopefully will give us more information [22]. Several studies focused at the effect of the route, dose, or timing of steroid administration. One small RCT study found no doseeffect of steroids on clinical outcomes [9]. Another RCT found no differences between the single intraoperative dose of steroid and double (pre-operative and intraoperative doses) [23]. One study investigated the effect of steroid administered intravenously versus steroid in prime and found no differences [24]. Due to the limitations of the randomised evidence described above, the resulting meta-analyses are of limited value too $[25,26]$. Some of the studies cited above focused on neonate patients only. This is because this group is particularly vulnerable to $\mathrm{CPB}$ insult due to (1) a distinct inflammatory response, (2) because of an immaturity of the HPA axis that prevents them to mount an adequate stress response. A recent systematic review of the literature found no clear benefit of steroid use in this particular group of patients [27].

The use of steroids will continue to be a matter of intense debate until we have the results of large, multicentre, randomised controlled trials that are able to detect any treatment effect in the context of congenital heart surgery that is performed nowadays with a very low morbidity and mortality. These trials will need to be similar in design to the large steroid trials in adult cardiac surgery [28].

\section{Are steroids worth the risk?}

Because of the lack of adequately powered studies, it is difficult to assess the short-term risk of steroid administration. However, several large registry retrospective studies of children undergoing heart surgery found no effect of steroids on 
clinical outcomes; furthermore, some raised concerns about infection in the steroid-treated groups [29-32]. Notably, the Pasquali et al. study retrospectively analysed a population of almost 46,730 children undergoing heart surgery and found no significant benefit of steroids. Furthermore, the steroids were associated with increased morbidity in the lower risk patient groups [29]. In other patient groups, there is emerging evidence that steroids have potential risks. A recently published retrospective study of more than half a million adults that received a short-term course of steroids found an increase in the rates of sepsis, venous thromboembolism, and fractures [33]. One RCT in children with premature lung disease found early administration of steroids to have a detrimental effect on neuromotor and cognition of outcomes if children were followed up to school age [34]. Another RCT in a similar group of patients with respiratory distress found steroids to be associated with increased incidence of cerebral palsy and developmental delay [35].Therefore, there is a need to design studies with long-term follow-up aimed at measuring effect of steroids on neurocognition.

\section{Steroids \\ and the hypothalamic-pituitary-adrenal axis}

In the context of congenital heart surgery, steroids are given not only to blunt inflammation but also as supplementation for what is called relative adrenal insufficiency [36]. The pathogenesis of this entity is indeed a matter of intense debate, and as a consequence, there are no agreed diagnostic criteria [36]. In the context of critical illness, most of the diagnostic tests rely on assessing the cortisol levels measured at a few time points and the response to a synthetic adrenocorticotropic hormone (ACTH) injection [37]. Nowadays, there is an increased recognition of the importance of biological clocks and hormone rhythms necessary for adequate physiological regulation [38]. Underlying the well-known circadian cortisol rhythm there is a fine, ultradian cortisol pulsatility [39]. Therefore, such frequent variations in the cortisol concentration make the ACTH tests inaccurate because they rely on a just few time-point measurements that can be taken either at the peak or trough of the pulse [40]. Several studies tried to assess the HPA axis during surgery [41-51]. Some used a few time cortisol measurements or just ACTH stimulation tests and tried to correlate their findings with clinical outcomes. In addition to limitations of the ACTH tests already described, some of these studies use variable ACTH test doses making interpretation more difficult [52]. Moreover, a major, common limitation of most of the studies that tried to characterise relative adrenal failure in children undergoing heart surgery is that these patients received steroids at induction making impossible an accurate assessment of the HPA axis.
Certainly, there is a need for studies aimed at understanding the basic HPA axis physiology during surgery. Such studies will need to be able to characterise cortisol pulsatility by using frequent cortisol measurements during the perioperative period, in children of various ages, that have not received any perioperative steroids. Once we understand HPA axis function during surgery, more studies aimed at the correlation of the hormone rhythms with clinical endpoints are required.

\section{Steroids, neuroprotection, and impact on neurocognitive outcomes}

Steroids are still used to treat brain oedema associated with brain tumours and were used for a long time in the treatment of head injuries until the large CRASH trial demonstrated that they can have detrimental effects [53-55]. Naturally, steroids are being used in cases with deep hypothermic circulatory arrest (DHCA) to provide neuroprotection [5]. However, the evidence in this area is only limited to in vitro [56] or in vivo, animal studies [57, 58]. Two studies used piglet models of DHCA arrest to assess the effect of steroids on brain injury. While one study found benefit [58], the other one found steroid administration to be detrimental [57]. These studies used different steroid regimens, DHCA experimental protocols, and methodologies to assess neuroprotection thus making difficult to draw any conclusions. The limited evidence in this high-risk group warrants the need for more clinical research powered to measure the effect of steroids on neuroprotection.

\section{Conclusions}

In the current review, we discussed the evidence for steroid use in paediatric cardiac surgery in the three main indication areas: effect on inflammation and clinical outcomes, the effect on adrenal function, and on neuroprotection. Due to the difficulty in characterising the complex inflammatory response to surgery, the current studies aimed to correlate the inflammatory effect of steroids with clinical outcomes remain limited. There is a lack of large randomised controlled trial powered to look at clinical outcomes. While there are some large registry studies looking at the effect of steroids on clinical outcomes, these remain limited by their retrospective design. Despite a wealth of data on the use of steroids in paediatric heart surgery, the current studies are conflicting and have many limitations thus leaving clinicians without any guidance. There is a limited understanding of the HPA axis physiology during the perioperative periods; therefore, we do not have an adequate diagnostic test to diagnose relative adrenal failure. We do not know if this entity even exists. Most of the studies aimed at assessing HPA axis during cardiac surgery are undertaken in patients already receiving steroids and use few cortisol 
measurements over long periods of times. Due to such limitations, we are unable to characterise the dynamic nature of the HPA axis response during the perioperative periods and to understand if steroids do play a role or not. The future research studies should aim at measuring cortisol levels at very frequent intervals to define the HPA axis function in the perioperative period. The least studied effect of steroids is on neuroprotection during DHCA surgery, and more research aimed at this high-risk group is needed.

Acknowledgements The study was supported by the British Heart Foundation and the NIHR Bristol Cardiovascular Biomedical Research Centre.

Funding information The study was supported by the British Heart Foundation and the NIHR Bristol Cardiovascular Biomedical Research Centre.

\section{Compliance with ethical standards}

Conflict of interest The authors declare that they have no conflict of interest.

Ethical approval All procedures performed in studies involving human participants were in accordance with the ethical standards of the institutional and/or national research committee and with the 1964 Helsinki Declaration and its later amendments or comparable ethical standards.

Informed consent Not required.

Open Access This article is distributed under the terms of the Creative Commons Attribution 4.0 International License (http:// creativecommons.org/licenses/by/4.0/), which permits unrestricted use, distribution, and reproduction in any medium, provided you give appropriate credit to the original author(s) and the source, provide a link to the Creative Commons license, and indicate if changes were made.

\section{References}

1. Cooley DA, Frazier OH. The past 50 years of cardiovascular surgery. Circulation [Internet]. American Heart Association, Inc.; 2000 [cited 2017 Nov 2];102:IV87-93. Available from: http://www.ncbi. nlm.nih.gov/pubmed/11080137.

2. Replogle RL, Gazzaniga AB, Gross RE. Use of corticosteroids during cardiopulmonary bypass: possible lysosome stabilization. Circulation. 1966;33:I86-92.

3. Laffey JG, Boylan JF, Cheng DC. The systemic inflammatory response to cardiac surgery: implications for the anesthesiologist. Anesthesiology. $2002 ; 97: 215-52$.

4. Nicholas Kouchoukos,Eugene Blackstone,Frank Hanley JK. Myocardial Management During Cardiac Surgery with Cardiopulmonary Bypass. Kirklin/Barratt-Boyes Card. Surg. Elsevier; 2012. p. 150-2.

5. Allen M, Sundararajan S, Pathan N, et al. Anti-inflammatory modalities: their current use in pediatric cardiac surgery in the United Kingdom and Ireland. Pediatr Crit Care Med. ; 2009;10:341-5.
6. Green ML, Koch J. Adrenocortical function in the postoperative pediatric cardiac surgical patient. Curr Opin Pediatr. 2012;24: 285-90.

7. Amanullah MM, Hamid M, Hanif HM, et al. Effect of steroids on inflammatory markers and clinical parameters in congenital open heart surgery: a randomised controlled trial. Cardiol Young. . 2016;26:506-15.

8. Bronicki RA, Backer CL, Baden HP, Mavroudis C, Crawford SE, Green TP. Dexamethasone reduces the inflammatory response to cardiopulmonary bypass in children. Ann Thorac Surg. 2000;69: $1490-5$.

9. Varan B, Tokel K, Mercan S, Dönmez A, Aslamaci S. Systemic Inflammatory Response Related to Cardiopulmonary Bypass and Its Modification by Methyl Prednisolone: High Dose Versus Low Dose. Pediatr Cardiol. 2002;23:437-41.

10. Lindberg L, Forsell C, Jogi P, Olsson AK. Effects of dexamethasone on clinical course, C-reactive protein, S100B protein and von Willebrand factor antigen after paediatric cardiac surgery. Br J Anaesth. 2003;90:728-32.

11. Heying R, Wehage E, Schumacher K, et al. Dexamethasone pretreatment provides antiinflammatory and myocardial protection in neonatal arterial switch operation. Ann Thorac Surg. 2012;93:869-76.

12. Schroeder VA, Pearl JM, Schwartz SM, Shanley TP, Manning PB, Nelson DP. Combined Steroid Treatment for Congenital Heart Surgery Improves Oxygen Delivery and Reduces Postbypass Inflammatory Mediator Expression. Circulation. 2003;107:2823-8.

13. Keski-Nisula J, Pesonen E, Olkkola KT, et al. Methylprednisolone in Neonatal Cardiac Surgery: Reduced Inflammation Without Improved Clinical Outcome. Ann Thorac Surg. 2013;95:2126-32.

14. Bocsi J, Hänzka M-C, Osmancik P, et al. Modulation of the cellular and humoral immune response to pediatric open heart surgery by methylprednisolone. Cytom Part B. 2011;80B:212-20.

15. Gessler P, Hohl V, Carrel T, et al. Administration of steroids in pediatric cardiac surgery: impact on clinical outcome and systemic inflammatory response. Pediatr Cardiol. 2005;26:595-600.

16. Jaffer U, Wade RG, Gourlay T. Cytokines in the systemic inflammatory response syndrome: a review. HSR Proc Intensive Care Cardiovasc Anesth. 2010;2:161-75.

17. Checchia PA, Bronicki RA, Costello JM, Nelson DP. Steroid use before pediatric cardiac operations using cardiopulmonary bypass: an international survey of 36 centers. Pediatr Crit Care Med. 2005;6:441-4.

18. Huber JN, Hilkin BM, Hook JS, et al. Neutrophil Phenotype Correlates With Postoperative Inflammatory Outcomes in Infants Undergoing Cardiopulmonary Bypass. Pediatr Crit Care Med. 2017;18:1145-1152.

19. Toledo-Pereyra LH, Lin CY, Kundler H, Replogle RL. Steroids in heart surgery: a clinical double-blind and randomized study. Am Surg. 1980;46:155-60.

20. Checchia Pa, Backer CL, Bronicki RA, et al. Dexamethasone reduces postoperative troponin levels in children undergoing cardiopulmonary bypass. Crit Care Med. 2003;31:1742-5.

21. Ando M, Park IS, Wada N, Takahashi Y. Steroid Supplementation: A Legitimate Pharmacotherapy After Neonatal Open Heart Surgery. Ann Thorac Surg. 2005;80:1672-8.

22. Graham EM. Corticosteroid Therapy in Neonates Undergoing Cardiopulmonary Bypass [Internet]. Available from: https:// clinicaltrials.gov/ct2/show/NCT01579513?term=steroid+ neonates\&rank $=3$

23. Graham EM, Atz AM, McHugh KE, et al. Preoperative steroid treatment does not improve markers of inflammation after cardiac surgery in neonates: Results from a randomized trial. J Thorac Cardiovasc Surg. 2014;147:902-8.

24. Keski-Nisula J, Suominen PK, Olkkola KT, et al. Effect of timing and route of methylprednisolone administration during pediatric cardiac surgical procedures. Ann. Thorac. Surg. 2015;99:180-5. 
25. Scrascia G, Rotunno C, Guida P, et al. Perioperative steroids administration in pediatric cardiac surgery: a meta-analysis of randomized controlled trials*. Pediatr Crit Care Med. 2014;15:435-42.

26. Robertson-Malt S, El Barbary M. Prophylactic steroids for paediatric open-heart surgery: a systematic review. Int J Evid Based Healthc. 2008;6:391-5.

27. Fudulu D, Schadenberg A, Angelini G, Stoica S. Perioperative use of steroids in neonatal heart surgery: Evidence based practice or tradition? Ann Med Surg. 2016;9:67-71.

28. Whitlock RP, Devereaux PJ, Teoh KH, et al. Methylprednisolone in patients undergoing cardiopulmonary bypass (SIRS): A randomised, double-blind, placebo-controlled trial. Lancet. 2015;386:1243-53.

29. Pasquali SK, Hall M, Li JS, et al. Corticosteroids and outcome in children undergoing congenital heart surgery: analysis of the Pediatric Health Information Systems database. Circulation. 2010;122:2123-30.

30. Pasquali SK, Li JS, He X, et al. Perioperative Methylprednisolone and Outcome in Neonates Undergoing Heart Surgery. Pediatrics. 2012];129:e385-91.

31. Dreher M, Glatz AC, Kennedy A, Rosenthal T, Gaynor JW. A Single-Center Analysis of Methylprednisolone Use during Pediatric Cardiopulmonary Bypass. J Extra Corpor Technol. 2015;47:155-9.

32. Elhoff JJ, Chowdhury SM, Zyblewski SC, Atz AM, Bradley SM, Graham EM. Intraoperative Steroid Use and Outcomes Following the Norwood Procedure: An Analysis of the Pediatric Heart Network's Public Database. Pediatr Crit Care Med. 2016;17:30-5.

33. Waljee AK, Rogers MAM, Lin P, et al. Short term use of oral corticosteroids and related harms among adults in the United States: population based cohort study. BMJ. 2017;357:j1415.

34. Yeh TF, Lin YJ, Lin HC, et al. Outcomes at School Age after Postnatal Dexamethasone Therapy for Lung Disease of Prematurity. N Engl J Med. 2004 ;350:1304-13

35. Shinwell ES, Karplus M, Reich D, et al. Early postnatal dexamethasone treatment and increased incidence of cerebral palsy. Arch Dis Child. Fetal Neonatal. 2000;83:177F-81.

36. Boonen E, Bornstein SR, Van den Berghe G. New insights into the controversy of adrenal function during critical illness. Lancet Diabetes Endocrinol. [Internet]. Elsevier Ltd; 2015;3:805-15.

37. Annane D, Sébille V, Troché G, Raphaël JC, Gajdos P, Bellissant E. A 3-Level Prognostic Classification in Septic Shock Based on Cortisol Levels and Cortisol Response to Corticotropin. JAMA.. 2000;283:1038-45.

38. The Nobel Assembly at Karolinska Institutet. The Nobel Assembly at Karolinska Institutet has today decided to award the 2017 Nobel Prize in Physiology or Medicine jointly to Jeffrey C . Hall, Michael Rosbash and Michael W. Young for their discoveries of molecular mechanisms controlling the circadian. 2017.

39. Spiga F, Walker JJ, Terry JR, Lightman SL. HPA axis-rhythms. Compr. Physiol. 2014;4:1273-98.

40. Gibbison B, Angelini GD, Lightman SL. Dynamic output and control of the hypothalamic-pituitaryadrenal axis in critical illness and major surgery. Br J Anaesth. 2013;111:347-60.

41. Kucera V, Hampl R, Stárka L. Corticoids during hypothermic openheart operations in children. Horm. Metab. Res. [Internet]. Germany; 1986;18:577-8.

42. Anand KJ, Hansen DD, Hickey PR. Hormonal-metabolic stress responses in neonates undergoing cardiac surgery. Anesthesiology. 1990;73:661-70.
43. Gajarski RJ, Stefanelli CB, Graziano JN, Kaciroti N, Charpie JR, Vazquez D. Adrenocortical response in infants undergoing cardiac surgery with cardiopulmonary bypass and circulatory arrest. Pediatr Crit Care Med. 2010;11:44-51.

44. Garcia X, Bhutta AT, Dyamenahalli U, Imamura M, Jaquiss RDB, Prodhan P. Adrenal insufficiency in hemodynamically unstable neonates after open-heart surgery. Congenit. Heart Dis. 2010;5:422-9.

45. Wald EL, Preze E, Eickhoff JC, Backer CL. The effect of cardiopulmonary bypass on the hypothalamicpituitary- adrenal axis in children. Pediatr Crit Care Med. 2011;12:190-6.

46. Verweij EJ, Hogenbirk K, Roest AAW, van Brempt R, Hazekamp $\mathrm{MG}$, de Jonge E. Serum cortisol concentration with exploratory cutoff values do not predict the effects of hydrocortisone administration in children with low cardiac output after cardiac surgery. Interact Cardiovasc Thorac Surg. 2012;15:685-9.

47. Schiller O, Dagan O, Birk E, et al. Adrenal insufficiency in children undergoing heart surgery does not correlate with more complex postoperative course. Pediatr Cardiol. 2013;34:1860-7.

48. Crow SS, Oliver WCJr, Kiefer JA, et al. Dexamethasone levels predict cortisol response after infant cardiopulmonary bypass. J Thorac Cardiovasc Surg. 2014;147:475-81.

49. Bangalore H, Ocampo EC, Rodriguez LM, et al. Serum cortisol and early postoperative outcome after stage-1 palliation for hypoplastic left heart syndrome. Pediatr Crit Care Med. 2014;15:211-8.

50. Crawford JH, Hull MS, Borasino S, et al. Adrenal insufficiency in neonates after cardiac surgery with cardiopulmonary bypass. Paediatr Anaesth. ; 2017;27:77-84.

51. Maeda T, Takeuchi M, Tachibana K, Nishida T, Kagisaki K, Imanaka H. Steroids Improve Hemodynamics in Infants With Adrenal Insufficiency After Cardiac Surgery. J Cardiothorac Vasc Anesth. 2016;30:936-41.

52. Graham EM, Bradley SM. First nights, the adrenal axis, and steroids . J Thorac Cardiovasc Surg. 2017;153: 1164-6.

53. Ryken TC, McDermott M, Robinson PD, et al. The role of steroids in the management of brain metastases: A systematic review and evidence-based clinical practice guideline. J Neurooncol. 2010;96: 103-14.

54. Baigent $\mathrm{C}$, Bracken $\mathrm{M}$, Chadwick D, et al. Final results of MRC $\mathrm{CRASH}$, a randomised placebocontrolled trial of intravenous corticosteroid in adults with head injury - outcomes at 6 months. Lancet. 2005;365:1957-9.

55. Muzha I, Filipi N, Lede R, et al. Effect of intravenous corticosteroids on death within 14 days in 10008 adults with clinically significant head injury (MRC CRASH trial): Randomised placebocontrolled trial. Lancet. 2004;364:1321-8.

56. Schmitt KRL, Kern C, Berger F, Ullrich O, Hendrix S, AbdulKhaliq H. Methylprednisolone attenuates hypothermia- and rewarming-induced cytotoxicity and IL-6 release in isolated primary astrocytes, neurons and BV-2 microglia cells. Neurosci Lett. 2006;404:309-14.

57. Schubert S, Stoltenburg-Didinger G, Wehsack A, et al. Large-Dose Pretreatment with Methylprednisolone Fails to Attenuate Neuronal Injury After Deep Hypothermic Circulatory Arrest in a Neonatal Piglet Model. Anesth Analg.2005;101:1311-8. 60. Langley SM, Chai PJ, Jaggers JJ, Ungerleider RM. Preoperative high dose methylprednisolone attenuates the cerebral response to deep hypothermic circulatory arrest. Eur J Cardiothorac Surg. ; 2000;17:279-86.

58. Langley SM, Chai PJ, Jaggers JJ, Ungerleider RM. Preoperative high dose methylprednisolone attenuates the cerebral response to deep hypothermic circulatory arrest. Eur J Cardiothorac Surg. ; 2000;17:279-86. 